\title{
On the Practicality of a Smart Contract PKI
}

\author{
Christos Patsonakis*, Katerina Samari*, Aggelos Kiayias ${ }^{\dagger}$ and Mema Roussopoulos* \\ *University of Athens, Greece, \{c.patswnakis,ksamari,mema\}@di.uoa.gr \\ ${ }^{\dagger}$ University of Edinburgh and IOHK, UK, Aggelos.Kiayias@ed.ac.uk
}

\begin{abstract}
Public key infrastructures (PKIs) are one of the main building blocks for securing communications over the Internet. Currently, PKIs are under the control of centralized authorities, which is problematic as evidenced by numerous incidents where they have been compromised. The distributed, fault tolerant $\log$ of transactions provided by blockchains and more recently, smart contract platforms, constitutes a powerful tool for the decentralization of PKIs. To verify the validity of identity records, blockchain-based identity systems store on chain either all identity records, or, a small (or even constant) sized amount of data to verify identity records stored off chain. However, as most of these systems have never been implemented, there is little information regarding the practical implications of each design's tradeoffs.

In this work, we first implement and evaluate the only provably secure, smart contract based PKI of [1] on top of Ethereum. This construction incurs constant-sized storage at the expense of computational complexity. To explore this tradeoff, we propose and implement a second construction which, eliminates the need for trusted setup, preserves the security properties of [1] and, as illustrated through our evaluation, is the only version with constant-sized state that can be deployed on the live chain of Ethereum. Furthermore, we compare these two systems with the simple approach of most prior works, e.g., the Ethereum Name Service, where all identity records are stored on the smart contract's state, to illustrate several shortcomings of Ethereum and its cost model. We propose several modifications for fine tuning the model, which would be useful to be considered for any smart contract platform like Ethereum so that it reaches its full potential to support arbitrary distributed applications.
\end{abstract}

\section{INTRODUCTION}

Contrary to its original, clean design principles, the Internet today is not completely decentralized. For example, Domain Name System (DNS) and Public Key Infrastructure (PKI) services, provide the most critical building blocks for facilitating and securing communications over the Internet. These systems manage mappings between identity names and values (i.e., an IP address for DNS, or a public-key for PKIs). Unfortunately, these critical systems are under the control of centralized, remote parties that must be trusted to function correctly. This is problematic as evidenced by the numerous incidents in, e.g., centralized PKIs, where certification authorities (CAs) have been compromised (e.g., [2], [3], [4]).

Since the advent of Bitcoin, blockchains show promise for building systems that are completely distributed with no trusted parties. Blockchains solve the well studied problem of distributed consensus ([5]) in an open networking environment. They provide a distributed, fault-tolerant, auditable, append-only ledger of transactions. As a result of this potential, there have been calls from the community to "re-decentralize" the Internet by leveraging blockchain technologies to build critical naming and PKI services and, thus, eliminate the Internet's reliance on centralized entities (e.g., [6], [7]).

Notable examples of blockchain-based identity management systems are Namecoin ([7]) and Emercoin ([8]). These systems employ the blockchain to store, verify and query for records pertaining to identities. However, this approach is inefficient for several reasons. First, it forces clients to download and maintain an entire copy of the blockchain to verify records. Second, computation and storage requirements scale linearly with the number of registered records. Third, it limits the system's applicability by excluding important, storage-limited devices, e.g., smartphones. Finally, it bloats the blockchain, increasing the size of the state that miners have to maintain, which may not align well with incentives of new miners that wish to sync and contribute to the blockchain's security.

To deal with these inefficiencies, researchers have recently proposed systems based on next-generation blockchains, i.e., smart contract platforms ([9], [1]). These systems decouple storage of identity records from their verification. Storage of identity records is handled by a separate, authenticated (but potentially unreliable) storage network which allows for a more compact retrieval of the full history of operations, compared to downloading and validating the entire blockchain. To verify the validity of identity records, they maintain on the smart contract's state cryptographic accumulators, which are space-efficient data structures that allow for verifiable membership and non membership queries.

Until now, these smart contract based identity management systems have not been implemented and, thus, there is little practical experience to guide developers and to inform design tradeoffs of future systems. In this paper, we examine the practical issues in building smart contract based PKI systems. We describe our experience of implementing on Ethereum the recently proposed, state-of-the-art, smart contract based PKI of Patsonakis et al. [1]. We choose this system because it is the only PKI with a security model and proof that it provides the claimed service while, at the same time, consuming constantsized state, as opposed to prior blockchain-based PKIs. The system's main building block is a public state, additive, universal accumulator based on the strong-RSA assumption. This accumulator favors storage overhead at the expense of computational overhead necessary to achieve constant-sized state and proofs. To explore the storage versus computational cost tradeoff, we propose a second smart contract based PKI built on top of the Hash tree-based, universal accumulator of [10]. Unlike the RSA-based accumulator, the Hash tree- 
based accumulator does not require a trusted setup and, thus, leads to a truly decentralized system while, at the same time, preserves the same security model and properties of the RSAbased approach. We also compare both of these systems with the simple approach of most prior schemes, e.g., the Ethereum Name Service ([11]), where all identity records are stored on the smart contract's state and we illustrate several shortcomings of Ethereum and its cost model.

In summary, the contributions of this paper are as follows. First, we evaluate experimentally all three smart contract based PKI schemes and illustrate the monetary costs of their operations, as well as those of their building blocks. Our results illustrate that the Hash tree-based construction is the only smart contract based PKI with constant-sized state that can be deployed on Ethereum's live chain, thus, providing the first viable and provably secure, on-blockchain authentication mechanism. Second, we present several shortcomings of Ethereum's current cost model and the ways in which: i) it affects each version of the PKI system, ii) it impedes the establishment of a standard library of smart contracts, iii) it incentivizes smart contract developers to adopt several malpractices and, iv) it prices on-blockchain storage of data. Third, we propose several modifications to Ethereum's cost model, which are minor and fair, to address the aforementioned issues and others. Finally, we identify several problem areas we encountered while developing on top of Ethereum and make concrete proposals for improving the platform with the aim of increasing both developer productivity and smart contract reliability. We argue that these improvements are sensible for any smart contract platform that wishes to support user developed distributed applications.

\section{RELATED WORK}

There is a large number of prior works on PKIs, in general. Due to lack of space, we review related work specifically on decentralized, blockchain and smart contract based PKIs.

Several works propose systems that employ the blockchain to store, query and verify the validity of each (identity,publickey) pair, or, some representation (e.g., hash) of it ([7], [8], [11], [12], [13], [14], [15], [16]). Blockstack ([6]) allows the development of arbitrary state machines via its virtualchains, i.e., it is a smart contract platform. Blockstack's BNS is a virtualchain (smart contract) that implements a distributed DNS. BNS stores the hashes of DNS zone files ([17]) on Atlas, Blockstack's distributed peer network. BNS clients verify the validity of each zone file hash stored in Atlas by searching the blockchain for a BNS transaction that contains this hash. Thus, BNS incurs the same inefficiencies of Namecoin and Emercoin, which we discussed in the Introduction. In contrast, our Hash tree-based PKI follows a different design principle that stores on the smart contract's state a constant and verifiable representation of all (identity,public-key) pairs by employing cryptographic accumulators. Clients of our PKI can verify the validity of records pertaining to identities via these accumulators and by interacting with a (potentially unreliable) storage network. Thus, in our PKI, there is no need to linearly search the blockchain. In addition, our design can be implemented on top of any system that allows the development of smart contracts. Thus, our Hash tree-based PKI can be even implemented on top of Blockstack. Nonetheless, we chose to implement our construction on top of Ethereum because it has a more rich and diverse ecosystem of applications. Multisignature wallets (e.g., [18]) and various (non) fungible tokens (e.g., [19]) are just a couple example applications that can benefit from the standard, on-blockchain authentication mechanism that our construction provides.

Melara et al. [20] introduce CONIKS, a privacy-preserving decentralized PKI where users can monitor the consistency of their own (identity,public-key) pairs. While privacy is an important property, e.g., for chat applications, it is not a requirement for traditional PKIs. For instance, in the web-PKI paradigm, the (identity,public-key) pairs of participants are public. CONIKS's operation is based on "identity providers", i.e., centralized entities that sign authenticated bindings and appropriately transform identity names for privacy purposes. CONIKS assumes the existence of a separate PKI to distribute the public keys of identity providers. Thus, it does not constitute a standalone PKI service, whilst our Hash tree-based PKI does. More importantly, CONIKS, lacks a formal proof of its security and privacy guarantees. This is also the case for systems derived from CONIKS, i.e., EthIKS ([21]), Catena ([22]) and Conifer ([23]), which implement CONIKS on top of Ethereum and Bitcoin. Certcoin ([9]) is a blockchain-based PKI proposal that employs cryptographic accumulators but has a number of inefficiencies, e.g., it recomputes, from scratch, accumulator values during each revocation. Furthermore, Certcoin has no security model for the PKI it implements nor a proof that it provides the claimed service ([1]).

Formal proofs of security are essential for critical security infrastructures. The Hash tree-based PKI we present here is built on top of the hash tree, universal accumulator of Camacho et al. [10], which is a public-state, additive, universal accumulator. Thus, it conforms to the design of the smart contract based PKI of Patsonakis et al. [1], which proposes the use of public-state additive, universal accumulators to realize a PKI and is the only PKI system with a formal proof of security. Furthermore, our construction advances the state-of-the-art system of Patsonakis et al. [1] by eliminating the need for a trusted setup phase and, thus, leads to a truly decentralized PKI service. Lastly, the state consumed by our construction is of constant size, contrary to systems such as EthIKS, whose state grows linearly with the number of identities registered in the system. We discuss the problems that arise from linear state (PKI) constructions on public smart contract platforms more thoroughly in Section VI-C

\section{ETHEREUM}

Ethereum is a blockchain-based platform for the development of smart contracts, i.e., stateful agents that "live" in the blockchain and can execute arbitrary state transition functions. Developing a smart contract involves writing its code in a high-level, Turing-complete programming language 
(e.g., Solidity [24]), which is then compiled-down to Ethereum Virtual Machine (EVM) initialization code. Contracts become part of Ethereum's global state (deployment) by wrapping their initialization code in a transaction, signing it and broadcasting it to the network. The state and the code of smart contracts are publicly accessible, thus, they can be trusted for correctness, provided their code was properly audited and the blockchain is secure, but not for privacy. Accessing the state of smart contracts can be performed efficiently via Ethereum's light client protocol $([25])$. Users can interact with contracts by issuing transactions that specify the code to be executed and its input arguments. In addition, contracts can call functions of other contracts, which is known as a message call, as a result of a user's transaction. Ethereum's cryptocurrency is called ether and serves as a means to incentivize participants (miners) to engage in the protocol. Transactions fees, which compensate miners for their work, are expressed in a unit called gas and are a function of their byte size and the complexity of the code they invoke (if any). Ethereum employs a flat cost model, i.e., each transaction byte and EVM operation costs some predefined amount of gas ([26]). Transactions specify a gas price, which converts ether to gas and influences the incentive of miners to include it in their next block. The higher the gas price, the higher its real monetary cost and priority to be mined ([27]). A transaction that consumes $g_{\text {cost }}$ gas and specifies a gas price of $g_{\text {price }}$ will cost $E=g_{\text {cost }} \times g_{\text {price }}$ units of ether. Lastly, transactions and message calls, specify an upper bound on the amount of gas that they can consume. This protects miners from, e.g., getting stuck in an infinite loop, an issue that stems from Ethereum's Turing-completeness.

\section{CRYptographic ACCumulators}

Cryptographic accumulators (first introduced in [28]) provide a constant-sized representation of a set of elements and allow for verifiable membership and, in some cases, non membership queries. In the design of [1], the smart contract acts as the accumulator manager and employs two instances of a public-state, additive, universal accumulator with domain $\{0,1\}^{*}$. First, as the smart contract's state and code are publicly accesible, the accumulator's operations have to be performed by exclusively relying on public information (public-state). Second, the accumulator's construction must, at least, support addition of elements (additive), to allow clients to register their (identity,public-key) pairs. Third, universal accumulators allow for both membership and non-membership verifications. Non membership verifications are essential since clients interested in registering to the PKI have to prove that their identity is not taken. In accumulators, proofs of, e.g., (non) membership, are referred to as witnesses.

The relevant syntax of the public-state, additive, universal accumulator of [1] to the constructions implemented in this work is:

- KeyGen $\left(1^{\lambda}\right)$ : Generates a key pair $(p k, s k)$ and outputs $p k$.

- InitAcc $(p k)$ : Outputs an accumulator value $c_{0}$, referring to the empty accumulated set $X \leftarrow \emptyset$.
- $\operatorname{Add}(p k, x, c)$ : Computes and outputs a new accumulator value $c^{\prime}$ and $W$, a membership witness for $x \in X$.

- MemWitGen $(p k, X, c)$ : If $x \in X$, it outputs a membership witness $W$ for $x$.

- NonMemWitGen $(p k, X, c, x)$ : If $x \notin X$, it outputs a non membership witness $W$ for $x$.

- VerifyMem $(p k, x, W, c)$ : If $W$ is an honestly produced membership witness for $x \in X$, it outputs 1 , otherwise, 0 .

- VerifyNonMem $(p k, x, W, c)$ : If $W$ is an honestly produced non membership witness for $x \notin X$, it outputs 1 , otherwise, 0 .

Below, we provide information on the specific accumulator constructions that we employ in our two implementations.

RSA-based Universal Accumulator: The construction of the smart contract based PKI of Patsonakis et al. [1] is built on top of the RSA-based universal accumulator of Li et al. [29]. This accumulator requires a trusted party to run the KeyGen algorithm since, knowledge of the accumulator's secret key $s k$ can be used to break its security. To deal with the issue that the accumulator's input domain is restricted to prime numbers, the authors of [1] incorporate a deterministic procedure Map that, on input an arbitrary string, outputs a prime number. This procedure is based on a function $f$, which is chosen uniformly at random from a universal hash function family $U:\{0,1\}^{3 k} \rightarrow\{0,1\}^{k}([30])$. An arbitrary string $s$ is mapped to a prime number by, first, computing the hash of the string, i.e., $h(s) \in\{0,1\}^{k}$, and by repeatedly sampling from the set $\left\{x \in\{0,1\}^{3 k}: f(x)=h(s)\right\}$ until a prime is found. The sampling is performed by fixing the randomness to depend on the input string. After sampling $O\left(k^{2}\right)$ times, Map will output a prime number, except with negligible probability. To circumvent the fact that deletions in RSA accumulators require access to the accumulator's secret key, the authors employ a trick that is presented in [31]. Essentially, the $i$-th time an input string $s$ is added or deleted, an element $x=(s, i, a)$ or $x=(s, i, d)$ is accumulated, respectively. Lastly, in the RSA accumulator, both the accumulator's value and its witnesses have constant bit size.

Hash tree-based Universal Accumulator: The accumulator of Camacho et al. [10], which our construction employs, is a public-state, additive, universal accumulator with the following differences and additional features. First and foremost, the accumulator is strong, i.e., the accumulator manager is not required to be trusted. Informally, a strong accumulator does not require a trusted setup (there is no KeyGen algorithm). Second, it supports the deletion of elements without relying on secret information. Third, it allows for additions/deletions which are publicly verifiable. The latter is accomplished by an algorithm CheckUpdate, which, on input a witness returned by either Add or Delete and the accumulator's values before and after the update, it outputs 1 , if the update was performed honestly and 0, otherwise. The accumulator of [10] is based on collision-resistant hash functions and its underlying data structure is a balanced, binary, hash tree. In a few words, the accumulator's value is the hash of the root node and witnesses 
are hash path(s) starting from some node(s) (not necessarily leaf node(s)) that lead all the way up to the root node. Note that the accumulator's value is of constant size, however, (non) membership and update witnesses have $\mathcal{O}(\lambda \log (n))$ bit size, where $n$ is the number of accumulated elements and $\lambda$ is the security parameter.

\section{Constructions}

In this section, we illustrate how we employ the constructs of Sections III and IV to realize two versions of the smart contract based PKI of [1]. The first is the construction provided in [1] and is based on the RSA accumulator that was highlighted in Section IV. The second, which we present in this work, is based on the Hash-tree accumulator of [10]. The core idea of both schemes is decoupling the storage of (identity,public-key) pairs from the verification of their validity.

The storage of information relevant to the protocol, e.g., (identity,public-keys) pairs, is offloaded to an external database component. In [1], this component is modeled as an unreliable database functionality $\mathcal{F}_{U D B}$ (referred to as $U D B$ from now on), since the adversary is allowed to arbitrarily modify its state. As proven in [1], the scheme's security is not affected by such adversarial behaviour. The involvement of $U D B$ in the protocol is twofold. First, clients query $U D B$ to obtain information that will allow them to, subsequently, interact with the smart contract. Second, following an interaction with the smart contract, the clients post to $U D B$ information that reflect the system's updated state.

The smart contract maintains two cryptographic accumulators to facilitate the verification of the validity of identities, or, (identity,public-key) pairs. The first accumulator, $c_{1}$, accumulates (identity,public-key) pairs, allowing clients to infer if a pair is currently registered or not. The second accumulator, $c_{2}$, accumulates identities, allowing clients to infer if an identity is registered or not. As shown in [1], one accumulator would suffice, however, at the expense of complicating the protocol's presentation and increasing its computational complexity, issues we also want to avoid here.

In both schemes, the smart contract is the most essential and expensive component to interact with in the system. Since its active involvement is required only during registration and revocation of (identity,public-key) pairs and due to lack of space, in this work, we only explain how these two operations are performed. To register her pair, a client queries the $U D B$ to obtain the history of operations, which will allow her to compute a proof that her identity is not accumulated in $c_{2}$. To revoke her pair, the client, instead, computes a proof that her pair is accumulated in $c_{1}$ and proves possession of the corresponding secret key. The latter is implemented via a digital signature on the public key.

\section{A. RSA-based PKI}

This construction is based on the RSA accumulator, which we highlighted in Section IV] Figure 3 of Appendix A illustrates the smart contract's pseudocode for this implementation.
Here, $c_{1}$ accumulates $(i d, p k, i, o p)$ tuples and $c_{2}$ accumulates $(i d, i, o p)$ tuples, where $o p=a$ or $o p=d$.

A client that wishes to register her (identity,public-key) pair produces, at most, two witnesses. First, a non membership witness $W_{1}$ for the tuple $(i d, i, a)$ in $c_{2}$. Second, and only if her identity has been registered at least once in the past $(i \geq 2)$, a membership witness $W_{2}$ for the tuple $(i d, i-1, d)$ in $c_{2}$. Assuming both conditions hold, she will be able to convince the smart contract that her identity is available. To construct these witnesses, the client queries $U D B$ for the history of operations and locates records (if any) pertaining to her identity to find the proper value for index $i$. Then, she invokes the Register function of the smart contract and, as a result, will receive the updated values of the accumulators and two new witness values, $W_{1}$ and $W_{2}$. These are membership witnesses for the tuples $(i d, p k, i, a)$ in $c_{1}$ and $(i d, i, a)$ in $c_{2}$, respectively. Next, the client computes a non membership witness $W_{3}$ for the tuple $(i d, i, d)$ in $c_{2}$. Lastly, she posts a (Register, $i d, p k, i, W_{1}, W_{2}, W_{3}$ ) record to the $U D B$ which, among others, facilitates queries from other clients for the validity of her mapping.

To revoke her (identity,public-key) pair, a client generates the following proofs. First, a signature of her public-key $\left(\sigma_{s k}(p k)\right)$. Second, a membership witness $W_{1}$ for the tuple $(i d, p k, i, a)$ in $c_{1}$. Third, a non membership witness $W_{2}$ for the tuple $(i d, p k, i, d)$ in $c_{1}$. The witnesses are constructed similarly to the case of registration, i.e., by querying $U D B$. Following a successful revocation of her mapping, the client posts a (Revoke, $i d, p k, i$ ) record to $U D B$.

\section{B. Hash tree-based PKI}

In this section, we propose an alternative construction, which is based on the Hash-tree accumulator of [10]. This accumulator supports additions and deletions which are publicly verifiable. Thus, clients can perform, locally, additions and deletions of elements and supply the smart contract with appropriate witnesses which prove that the operations were performed honestly. To generate all involved witnesses, clients query $U D B$ for the history of operations. Figure 4 of Appendix Aillustrates the smart contract's pseudocode for this implementation.

To register an (identity,public-key) pair, the client generates a non membership witness $W_{2}$ for her identity in $c_{2}$. She then performs, locally, the following updates. First, she accumulates the tuple $(i d, p k)$ in $c_{1}$, which produces an addition update witness $W_{a d d_{1}}$ and the updated value of the accumulator $c_{a d d_{1}}$. Second, she accumulates her identity in $c_{2}$, which produces an addition update witness $W_{a d d_{2}}$ and the accumulator's updated value $c_{a d d_{2}}$. Assuming all values were computed honestly, the contract will validate the proofs by invoking CheckUpdate $\left(c_{2}, c_{a d d_{2}}, W_{a d d_{2}}, i d\right)$, CheckUpdate $\left(c_{1}, c_{a d d_{1}}, W_{a d d_{1}},(i d, p k)\right)$ and VerifyNonMem $\left(c_{2}, W_{2}, i d\right)$, and update its accumulator values. Lastly, the client posts a (Register, $i d, p k)$ to the $U D B$.

To revoke an (identity,public-key) pair, the client first signs her public-key $\left(\sigma_{s k}(p k)\right)$. Second, she generates a membership 
witness $W_{1}$ for the tuple $(i d, p k)$ in $c_{1}$. She then performs, locally, two updates. First, she deletes her identity from $c_{2}$, which produces a deletion update witness $W_{d e l_{2}}$ and the accumulator's updated value $c_{d e l_{2}}$. Second, she deletes the tuple $(i d, p k)$ from $c_{1}$, which produces a deletion update witness $W_{d e l_{1}}$ and the accumulator's updated value $c_{d e l_{1}}$. Assuming all values were computed honestly, the contract will validate the proofs by invoking CheckUpdate $\left(c_{1}, c_{d e l_{1}}, W_{d e l_{1}},(i d, p k)\right)$, CheckUpdate $\left(c_{2}, c_{d e l_{2}}, W_{d e l_{2}}, i d\right)$ and $\operatorname{Verify} \operatorname{Mem}\left(c_{1}, W_{1},(i d\right.$ $, p k)$ ), and update its accumulator values. Lastly, the client posts a (Revoke, $i d, p k$ ) record to the $U D B$, which is not stored, but simply leads to the deletion of her registration record.

\section{EVALUATION}

In this section, we present experiments that measure the cost of running on Ethereum the constructions of Section $\nabla$ as well as their building blocks. Throughout this section, we intersperse our results with recommendations for modifications and/or improvements to Ethereum that, we believe, are vital if Ethereum (or any smart contract platform) is to reach its maximum potential of supporting arbitrary distributed applications (especially in the large scale [32]). We create a private blockchain that is maintained by a single mining node. This eliminates the waiting time that transactions would have in either the live or the test chain to be mined into a block. Thus, we are able to measure accurately transaction gas costs and perform experiments on a larger scale. We run our experiments on a CentOS 7 server that is equipped with an 8-core, 64-bit, Intel(R) Xeon(R) CPU E5-2620 v4 @ $2.10 \mathrm{GHz}$ (with hyperthreading) and $32 \mathrm{~GB}$ of RAM. We use the latest, stable release of geth (v.1.8.17, [33]), the official Ethereum client. We conduct our experiments via the truffle suite (v.4.1.13, [34]), a testing framework that automatically handles compilation and deployment of contracts and provides easy-to-use, JavaScript-based means of interacting with them. Finally, we use randomly generated 32-byte identities.

Our implementations employ a variety of primitives, e.g., signatures and accumulators. One option would be to deploy each primitive as a separate library and have the front-end PKI contract issue appropriate message calls. Unfortunately, this option is the most expensive in terms of gas due to the extra cost of message calls (700 gas) and the increased cost of reading the deployment address(es) of the library contract(s) from storage. The more efficient option is to pack all backend logic into a single library and link it with the front-end PKI contract. This eliminates the aformentioned costs. Thus, Ethereum imposes the following tradeoff. On the one hand, developers will tend to pick the second option, as one of their main incentives is to minimize gas cost. On the other hand, the first option: 1) promotes modular programming, 2) leads to the construction of an on-blockchain "standard library", similar to what common programming languages have and most importantly, 3) mitigates duplicate logic, i.e., excess, duplicate state and code in the blockchain. Thus, reducing the costs of the first option will aid in the development of future applications and incentivize developers to adopt more modular programming approaches.

Recommendation \#1: Significantly reduce the cost of issuing message calls to libraries.

Our first experiment provides insight regarding the overhead of a library implementation, compared to a precompiled contract. Precompiled contracts reside on well-known, static addresses and require less gas because their code does not run in EVM assembly, but in machine language of the physical node hosting the miner. We evaluate the cost of verifying 1,000 Secp256k1 elliptic curve signatures, based on the library contract of [35]. We measure a mean cost of 827,765.53 gas, with a standard deviation of $6,021.64$ gas. At the time of this writing, the average gas limit of blocks is about 8 million gas ([36]). Thus, signature verification on the library contract consumes $10.3 \%$ of the current block gas limit, which is substantial. In contrast, Ethereum's ecrecover precompiled contract, which operates on the same curve, costs only 3,000 gas. Thus, the cost of the library implementation is two orders of magnitude higher, which illustrates the benefits and importance of having built-in support for a variety of cryptographic operations. In the evaluation of all the constructions that follow, we have modified the library contract of [35] to operate on the Secp256r1 curve. We repeat the same experiment and measure the mean cost of signature verification to be $1,257,103.26$ gas, with a standard deviation of 9,178.44 gas.

\section{A. RSA-based PKI Evaluation}

In this section, we evaluate the RSA-based PKI of Section $\mathrm{V}$-A which employs the following constructs. First, signature verification via the Secp256r1 library contract. Second, arbitrary precision integer arithmetic, based on the Big Number library developed by the Zerocoin team ([37]). This library supports operations which are relevant to this construction, such as modulo exponentiation and the Miller-Rabin probabilistic primality test. We modify the implementation of the primality test because: 1) the original supports only a range of integers, whilst, our implementation supports all integers, 2) the original algorithm is seeded by externally provided randomness, which we modify to be based on the hash of the last block, thus, limiting the adversary's knowledge and influence on its output and, 3) the original does not perform sufficient iterations, which we modify to comply to the NIST standard ([38]), i.e., 64 witness loop iterations, thus, the probability a composite number will be declared as prime is $2^{-128}$. The third employed construct is the RSA accumulator, which encompasses the Map procedure. The RSA moduli of the accumulators are 3072 bits long, thus, they provide 128-bit security ([39]). Recall that Map uses a function $f:\{0,1\}^{3 k} \rightarrow\{0,1\}^{k}$ chosen uniformly at random from a universal hash function family $U$. We set Map's parameter to $k=65$, i.e., Map outputs $3 k=195$ bit primes. Thus, except for $1 / 2^{65}$ fraction of functions $f \in U$, a string will be mapped to a prime number, except with negligible probability ([40]), which we deem reasonable. 


\begin{tabular}{|r|r|r|r|r|}
\hline \multirow{2}{*}{ Operation } & \multicolumn{4}{|c|}{ Gas Cost } \\
\cline { 2 - 5 } & Min & Max & Mean & Std \\
\hline Mod. Mul. & 179,556 & 182,900 & $181,470.76$ & 639.16 \\
\hline Mod. Exp. & 678,074 & 745,517 & $741,846.48$ & $5,001.49$ \\
\hline Primality Test & $1,481,160$ & $1,502,502$ & $1,490,219.13$ & $5,480.23$ \\
\hline Add & 810,030 & 810,158 & $810,153.32$ & 17.61 \\
\hline VerifyMem & 755,130 & 755,796 & $755,537.23$ & 126.63 \\
\hline VerifyNonMem & $1,473,345$ & $1,525,685$ & $1,519,279.96$ & $5,386.87$ \\
\hline Map & $1,733,124,331$ & $2,550,435,741$ & $2,141,780,036$ & $577,926,440.35$ \\
\hline Register $(i=1)$ & $89,801,425$ & $8,620,016,945$ & $1,681,994,990$ & $1,313,539,096.67$ \\
\hline Register $(i \geq 2)$ & $89,160,026$ & $10,676,126,282$ & $2,575,538,734.5$ & $1,715,254,997.91$ \\
\hline Revoke & $440,467,878$ & $13,805,874,517$ & $3,598,585,618$ & $1,910,918,965.1$ \\
\hline
\end{tabular}

TABLE I: Min, max, mean and standard deviation (columns 2-5) of the gas cost of: 1) 10,000 modulo multiplications, exponentiations and primality tests in the Big Number library, 2) 10,000 accumulations of primes (Add) and (non) membership witness verifications (VerifyMem, VerifyNonMem) 3) 1,000 mappings (Map) of strings to primes and, 4) registrations (Register, for $(i=1)$ and $(i \geq 2)$ ) and revocations (Revoke) of 1,000 (identity,public-key) pairs in the RSA-based PKI.

We conduct four sets of experiments where the bit lengths of the exponents, moduli and exponentiation bases are 195, 3072 and 3072 bits long, respectively. First, we evaluate the operations of the Big Number library that are relevant to this construction by running 10,000 primality tests, modulo multiplications and exponentiations, respectively. Second, we evaluate the RSA accumulator by running 10,000 iterations of each of the following operations: 1) accumulations (Add) of 195-bit prime numbers, and 2) (non) membership witness verifications (VerifyMem, VerifyNonMem). Third, we measure the cost of 1,000 mappings (Map) of strings to 195-bit prime numbers. Fourth, we measure the cost of registering (Register) and revoking (Revoke) 1,000 (identity,public-key) pairs in the RSA-based PKI. Recall that registration differentiates between two cases, i.e., whether an identity is registered for the first time $(i=1)$, or not $(i \geq 2)$. Table $\prod$ illustrates the results.

Regarding the Big Number library experiments, Table II shows that modulo exponentiation and primality testing are the more expensive operations. The former is based on one of Ethereum's precompiled contracts ([41]) and its cost is dominated by the exponent's length, especially in cases where it is larger than 32 bytes. In addition, this operation is invoked in the (main) witness loop of the Miller-Rabin test, thus, the data suggest that, on average, the primality test performs two loop iterations. To compute the cost of the RSA accumulator's operations, we have to factor in the cost of reading from storage the accumulator's value, its exponentiation base and its modulus (a total of $36 \mathrm{EVM}$ words). The cost of reading an EVM word (32 bytes) from storage is 200 gas, thus, $36 \times 200=7,200$ extra gas. The VerifyMem operation involves one modulo exponentiation. The VerifyNonMem operation involves two modulo exponentiations and one modulo multiplication. Thus, as the data suggest, the gas cost of these two operations follows directly from that of reading the appropriate values from the contract's storage and the invoked operations of the Big Number library. The Add operation involves one modulo exponentiation and modifies the accumulator's value. Thus, in addition to the aforementioned cost of reading from the contract's storage, there is also the cost of storing the accumulator's updated value to the contract's state. Updating an EVM word on storage costs 5,000 gas. The accumulator is 12 EVM words long, thus, $12 \times 5,000=60,000$ extra gas, or, a total of $60,000+7,200=67,200$ gas.

The key result of this section is our demonstration of the practical implications of the RSA-based PKI's design. Recall that in this construction, the smart contract's state and (non) membership witnesses have constant size at the expense of computational overhead. This tradeoff is embodied by the $O\left(k^{2}\right)$ complexity of the Map procedure, which is involved in both the Register and Revoke operations of the RSAbased PKI, to map the contract's inputs to prime numbers. These prime numbers are then input to the appropriate witness verification algorithms of the RSA accumulator. For instance, the Revoke operation involves one signature verification, one invocation of VerifyNonMem and VerifyMem each and three invocations of Map. We have already illustrated that the cost of signature verification and of the RSA accumulator's operations is deterministic, however, the same cannot be said about Map. While Map is deterministic in terms of its output, the number of iterations it performs to produce its output is not. Thus, we can have cases where one invocation of Map costs more than a Register or Revoke operation of the RSA-based PKI, as illustrated by the heavily skewed data of Table Consequently, Map dominates the cost of the RSA-based PKI's operations. We perform an additional experiment where we measure the cost of running one iteration of Map on input of 100,000 strings. We measure the average gas cost to be $9,488,542.32$ gas, with a standard deviation of 17,794.86 gas. Consequently, even one iteration of Map exceeds Ethereum's block gas limit.

Result \#1: The provably secure, RSA-based smart contract PKI is not viable in Ethereum.

Discussion: There are two reasons why Map's gas cost is so high. First, in Ethereum, it is cheaper to access one EVM word (32 bytes) than one byte. This "was chosen to facilitate the Keccak256 hash scheme and elliptic-curve computations", as stated in Ethereum's yellow paper ([26]). It has nothing to do with efficiency as no real-world physical machine, on top of which the EVM runs, supports 32 byte words. Therefore, one potential improvement would be to modify Ethereum's cost model to account for this contradiction. For instance, accessing 
a single byte could be simply tuned to $\frac{1}{32}$ of the cost of loading an EVM word. This change, apart from being more fair, allows for more packed data encodings which can reduce the size of transactions and, as a result, the size of the blockchain. Second, Map's computation revolves around bit operations, which are, currently, very expensive in Ethereum as they have to be performed via the EVM's integer exponentiation function. For instance, setting one bit of a memory byte array costs 586 gas. However, in the near future, the EVM will support bitwise shifting ([42]), which will only cost 3 gas and, thus, will provide substantial improvements for Map.

Recommendation \#2: Ethereum's cost model should be modified to account for the granularity of the data that are accessed.

Nevertheless, for the RSA-based PKI, there are other alternatives that we can explore, which we leave as future work, that will also benefit from all the aforementioned propositions, such as verifiable computation ([43]). In this setting, the smart contract will need only to verify proofs that the client computed Map correctly, instead of invoking Map itself.

\section{B. Hash tree-based PKI Evaluation}

In this section, we evaluate the Hash-based PKI, which we proposed in Section $\mathrm{V}-\mathrm{B}$. This construction employs the following primitives: 1) the Secp256r1 library contract for signature verification and, 2) the Hash tree accumulator of Section IV] We employ the SHA-256 hash function, which is exposed as a precompiled contract in Ethereum. Recall that in the Hash-based PKI, all involved proofs are a logarithmic function of the number of accumulated elements, in contrast to the RSA-based construction, where they are constant-sized.

In our first experiment, we accumulate a total of 100,000 elements and measure the cost of the Hash tree accumulator's operations. In Figures $1 \mathrm{a}$ and $1 \mathrm{~b}$, we plot the gas cost versus the number of accumulated elements of verifying (non) membership witnesses (VerifyMem, VerifyNonMem) and accumulator updates (CheckUpdate), respectively. The general trend resembles, as expected, that of a logarithmic function. However, the curves have a large number of dips. This is because in this scheme, proofs are paths of the accumulator's hash tree. The size of each proof depends on the position of the starting node(s) in the hash tree and, thus, its verification cost varies. As illustrated in the graphs, verifying accumulator updates is more expensive than that of (non) membership witnesses. Indeed, the size of the former proofs tends to be two, or even, three times the size of the latter, which is reflected in their respective verification costs.

In our second experiment, we evaluate our Hash-based PKI construction. In Figure 2, we plot the gas cost versus the number of registered pairs of registering and revoking 100,000 (identity,public-key) pairs. The results show that revocation is the more costly procedure as it involves the added cost of verifying signatures. The cost of the most expensive revocation that we measured was 2,999,214 gas, i.e., $37.4 \%$ of the current block gas limit. Thus, in terms of gas cost, this construction can be deployed on the main chain of Ethereum.
Result \#2: Our Hash-based PKI construction is viable for deployment on the main chain of Ethereum.

Discussion: The Hash-based PKI is best suited for small, to moderately sized PKIs, since the involved proofs are not of constant size. Assuming that the number of registered pairs monotonically increases, there will come a point where verifying proofs will exceed the block's gas limit. Recall that the cost of a transaction is a function of its computational complexity and its byte size. One might argue that this issue can be balanced out by an increase in the block's gas limit, which is certainly the observed trend up to the time of this writing ([36]). However, a miner's main incentive is to produce (hash) blocks as fast and with as low operational costs as possible. Thus, it can be expected that the increase in the block's gas limit will, eventually, plateau. This line of reasoning assumes that the blockchain's consensus mechanism revolves around Proof-of-Work (PoW), as is currently the case. However, Ethereum is planning to replace PoW with Proof-of-Stake $(\mathrm{PoS})$, a consensus protocol that requires a small amount of computation. Discussing PoS is out of the scope of this paper, however, it is reasonable to assume that it will change the incentives of miners. Indeed, in this computationally light paradigm, miners might be willing to expend their computational resources to mine blocks that contain larger transactions to maximize their rewards. This will gradually increase the block's gas limit which, as a result, will favor the scale of our Hash tree-based PKI even more.

We now illustrate a few important points regarding hash functions and precompiled contracts. Ethereum supports three hash functions: 1) RIPEMD160, whose computation costs 600 gas, plus 120 gas per input word, 2) SHA-256, whose computation costs 60 gas, plus 12 gas per input word, 3) KECCAK-256, whose computation costs 30 gas, plus 6 gas per input word. Functions (1) and (2) comply with the NIST standard and are implemented as precompiled contracts, however, function (3), does not comply with the standard and is implemented as an EVM opcode. These distinctions have interesting implications. Because functions (1) and (2) are precompiled contracts, they incur the extra gas cost of a message call (700 gas), while function (3) does not. Consequently, Ethereum's cost model encourages the use of a non-standard-compliant hash function. Thus, application developers are forced to either code the client side (at least in part) in JavaScript, for the sole purpose of having access to Ethereum's non-standard implementation of (3), or, pay the extra gas cost. As one of the main incentives of developers in these platforms is to minimize gas costs, the aforementioned distinctions essentially encourage client implementations that are unnecessarily complicated and limit the use of standard, mature and efficient libraries, such as libgcrypt ([44]). Recently, a proposal has been submitted ([45]) to address this issue. If accepted, this change will further diminish the gas cost of the Hash treebased PKI, thus, increasing its deployment scale.

Recommendation \#3: Reduce message call costs from precompiled contracts and equalize the costs of all supported hash functions. 


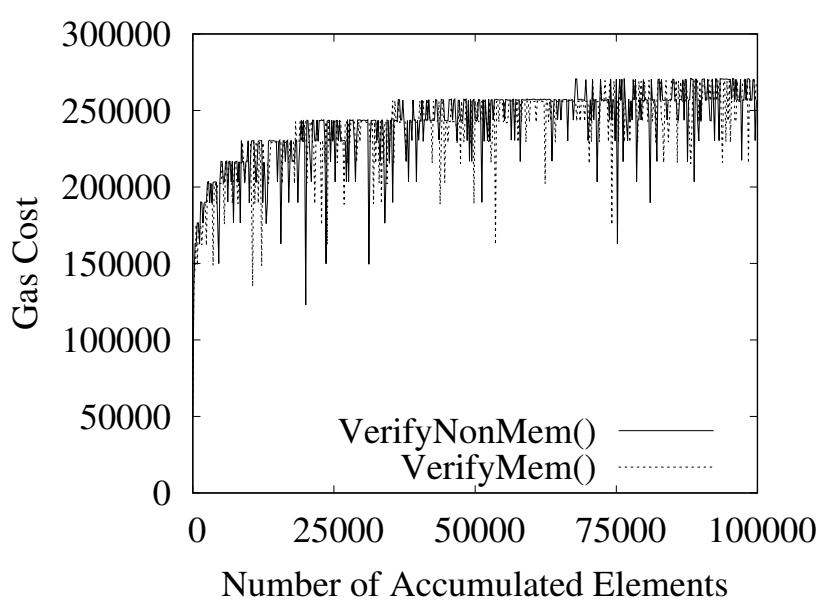

(a)

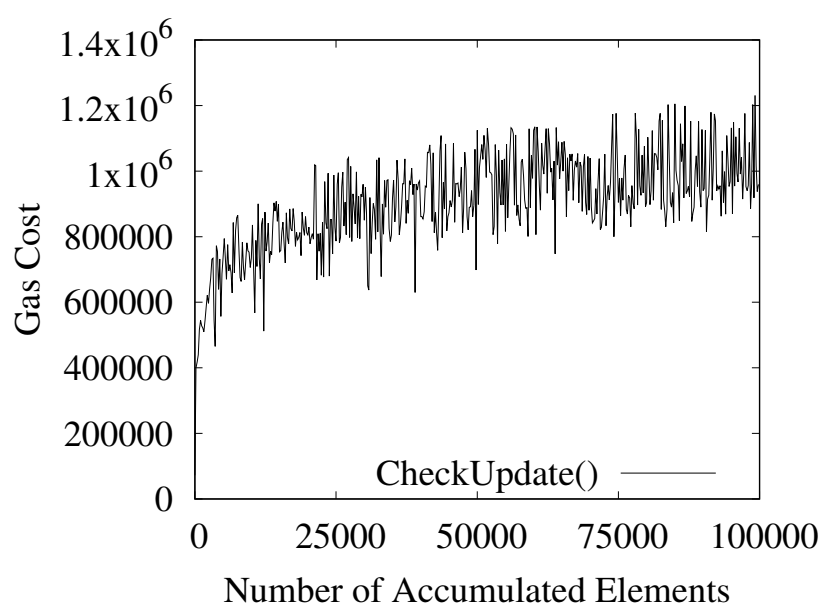

(b)

Fig. 1: Gas cost versus the number of accumulated values of 100,000 of the following hash tree accumulator verifications: (a) (Non) Membership witnesses and, (b) Accumulator updates. Note the different y-axis scales.

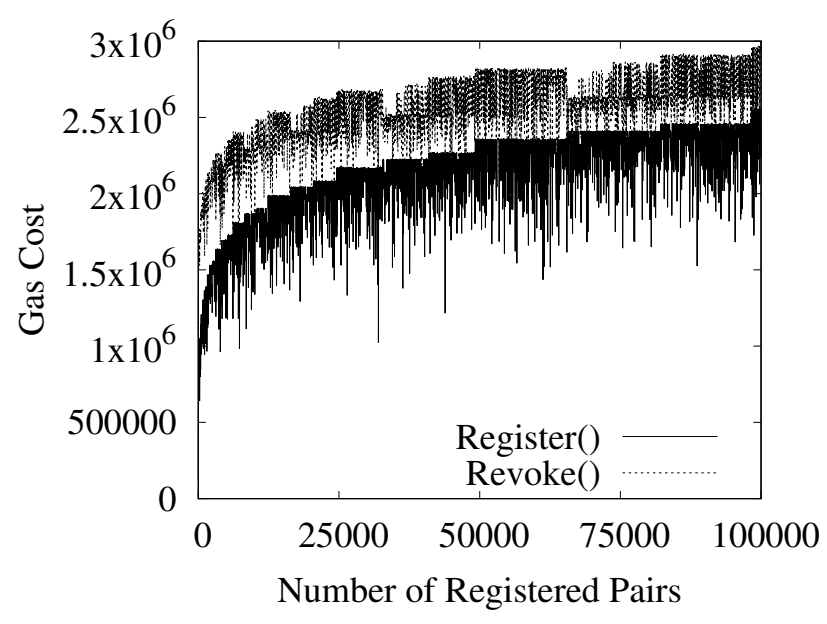

Fig. 2: Gas cost versus the number of registered (identity,public-key) pairs of the registration and revocation operations of 100,000 pairs in the Hash-based PKI.

Lastly, as illustrated previously, Ethereum is inconsistent in the way it handles and exposes more complicated instructions. Given that: 1) the size of EVM assembly opcodes is one byte, 2) most values are already in use ([46]) and, 3) the purpose of assembly language is to provide access to low-level instructions, we believe that more sophisticated functionality (e.g., hash functions) should be offloaded to a standard library of precompiled contracts. Furthermore, Ethereum should design and incorporate a more developer-friendly way of addressing these contracts. Currently, developers need to memorize (or look up) the address in which each contract resides, e.g., SHA256 resides in address $0 x 02$, which is cumbersome. Convenient helper functions in EVM assembly that are translated to the appropriate message calls would be helpful.

Recommendation \#4: Sophisticated functionality should

\begin{tabular}{|c|c|c|c|c|}
\hline \multirow{2}{*}{ Operation } & \multicolumn{4}{|c|}{ Gas Cost } \\
\cline { 2 - 5 } & Min & Max & Mean & Std \\
\hline Register & 89,469 & 89,661 & $89,643.91$ & 33.74 \\
\hline Revoke & 904,197 & 949,505 & $931,150.28$ & $6,410.29$ \\
\hline
\end{tabular}

TABLE II: Min, max, mean and standard deviation (columns $2-5$ ) of the gas cost of registering and revoking 10,000 randomly generated (identity,public-key) pairs in the Linear State PKI contract.

be moved to a standard library of precompiled contracts that can be addressed in a developer-friendly manner.

\section{Linear State PKI Evaluation}

In this section, we present experiments that evaluate a simple smart contract PKI which stores all (identity,publickey) pairs in the contract's state. This is the same approach that prior proposals employ (e.g., [11], [12], [13]), including the Ethereum Name Service, and allows us to illustrate the shortcomings of Ethereum's pricing of storage. In this simple scheme, registration and revocation are straightforward processes. During registration, the contract checks if there is an entry for the input identity in its state. Assuming there isn't one, it adds it. During revocation, the contract first validates the input signature, as in the prior two constructions, and checks if there is an entry in its state for that identity. If so, the contract simply removes it from its state. Table 1 illustrates the gas cost of registering and revoking 10,000 (identity,public-key) pairs in this setting. During revocation, part of the contract's storage is freed, and the transaction is refunded gas. As a result, the overall cost of revocation is less than the verification of a Secp256r1 signature.

Discussion: Clearly, this is, currently, the least costly approach to deploy on Ethereum and the reasons are straightforward. First, it is light in terms of computation. Indeed, excluding signature verification, which is the dominant cost 
of revocation, the contract spans a total of 10 lines of Solidity code, consisting solely of a few if statements. Second, as stated by Buterin $([47])$, storage is extremely underpriced and, as of yet, there is no incentive for freeing it. However, the issue of storage and its effect on the size of Ethereum's state is complex. Miners decide whether they include a transaction in a block according to their "private cost", i.e., their own private resource expenditure. Regardless, their decision affects the entire network, as all participating nodes have to download and validate newly mined blocks, without having a choice in the matter. For instance, if we take the Linear State PKI as a reference point, it might be favorable for some miner(s) to store a few bytes on disk, e.g., due to having abundant and inexpensive disk space. However, that may not be the case for other nodes participating in the protocol. This suggests a "social cost" of transactions which may not be completely aligned to an individual miner's private costs. If this social cost of transactions is not completely accounted for, the increasing size of Ethereum's state may deincentivise new full nodes from entering the system. Furthermore, the size of Ethereum's state can also be an obstacle to nodes merely syncing with the system. This issue affects a variety of topics ranging from, light clients (e.g., smartphones) being able to interface with smart contracts, to blockchain security.

One of the proposed countermeasures is imposing small, static rent fees on contracts ([48]) so as to avoid being "deactivated", i.e., no one being able to interact with them. However, it is Ethereum's static cost model that has caused this problem in the first place. We believe storage is a special commodity and that its price should be dynamically adjusted. Base storage price should depend on the global size of Ethereum's state, i.e., the bigger the size of its state, the higher the base storage price. In addition, the cost of transactions that increase the size of a contract's state should scale accordingly, thus, providing a counterincentive to over-utilizing contract storage.

Recommendation \#5: Storage costs should take into account the current blockchain's size as well as the size of the invoked contract's state.

Clearly, the issue of storage costs is complex and remains an open problem requiring future research to be addressed properly. Nonetheless, the constant state PKI constructions discussed in this work are well aligned with space optimal use of smart contract platforms.

\section{REFERENCES}

[1] C. Patsonakis, K. Samari, M. Roussopoulos, and A. Kiayias, "Towards a smart contract-based, decentralized, public-key infrastructure," in 16th International Conference on Cryptology and Network Security (CANS), 2017.

[2] "Final report on diginotar hack shows total compromise of ca servers," https://tinyurl.com/hnmuahc

[3] "Google takes symantec to the woodshed for mis-issuing 30,000 https certs," https://tinyurl.com/y8y4qpfx

[4] "Trustwave admits it issued a certificate to allow company to run manin-the-middle attacks," https://tinyurl.com/ycfv6kfs

[5] L. Lamport, R. Shostak, and M. Pease, "The byzantine generals problem," ACM Trans. Program. Lang. Syst., 1982.

[6] M. Ali, J. Nelson, R. Shea, and M. Freedman, "Blockstack: A global naming and storage system secured by blockchains," in USENIX Annual Technical Conferencei (ATC), 2016.
[7] "Namecoin," https://namecoin.org/

[8] "Emercoin," http://www.emercoin.com

[9] C. Fromknecht, D. Velicanu, and S. Yakoubov, "A decentralized public key infrastructure with identity retention," IACR Cryptology ePrint Archive, 2014.

[10] P. Camacho, A. Hevia, M. A. Kiwi, and R. Opazo, "Strong accumulators from collision-resistant hashing," in Information Security, 11th International Conference (ISC), 2008.

[11] "Ethereum name service," https://ens.domains/

[12] M. Al-Bassam, "Scpki: A smart contract-based pki and identity system," in Proceedings of the ACM Workshop on Blockchain, Cryptocurrencies and Contracts, 2017.

[13] A. Yakubov, W. M. Shbair, A. Wallbom, D. Sanda, and R. State, "A blockchain-based pki management framework," in NOMS 2018 - 2018 IEEE/IFIP Network Operations and Management Symposium, 2018.

[14] S. Matsumoto and R. M. Reischuk, "Ikp: Turning a pki around with decentralized automated incentives," in IEEE Symposium on Security and Privacy (SP), 2017.

[15] Q. C. Q. W. J. J. Ze Wang, Jingqiang Lin and D. Zha, "Blockchain-based certificate transparency and revocation transparency," in 5th Workshop on Bitcoin and Blockchain Research, 2018.

[16] Z. Q. Junjun Lou, Qichao Zhang and K. Lei, "A blockchain-based key management scheme for named data networking," in IEEE International Conference on Hot Information-Centric Networking (HotICN), 2018.

[17] "Ietf: Domain names - implementation and specification," https://tools.ietf.org/html/rfc1035

[18] "Consensys: Ethereum multisigwallet," https://tinyurl.com/y8kxz78u

[19] "Erc20 token market capitalization," https://etherscan.io/tokens

[20] M. S. Melara, A. Blankstein, J. Bonneau, E. W. Felten, and M. J. Freedman, "CONIKS: Bringing key transparency to end users," in 24th USENIX Security Symposium (USENIX Security), 2015.

[21] J. Bonneau, "Eth iks: Using ethereum to audit a coniks key transparency log," in Financial Cryptography and Data Security - International Workshops, FC 2016, BITCOIN, VOTING, and WAHC, 2016.

[22] A. Tomescu and S. Devadas, "Catena: Efficient non-equivocation via bitcoin," in IEEE Symposium on Security and Privacy (SP), 2017.

[23] W. K. Y. Dong and R. Boutaba, "Conifer: centrally-managed pki with blockchain-rooted trust," in IEEE International Conference on Blockchain (Blockchain), 2018.

[24] "Solidity," https://tinyurl.com/yae73x2n

[25] "Ethereum light client protocol," https://tinyurl.com/zha9w8p

[26] G. Wood, "Ethereum yellow paper," https://tinyurl.com/yaptyawg

[27] "Eth gas station," https://ethgasstation.info/

[28] J. C. Benaloh and M. de Mare, "One-way accumulators: A decentralized alternative to digital sinatures (extended abstract)," in Advances in Cryptology - (EUROCRYPT), 1993.

[29] J. Li, N. Li, and R. Xue, "Universal accumulators with efficient nonmembership proofs," in 5th International Conference on Applied Cryptography and Network Security (ACNS), 2007.

[30] L. Carter and M. N. Wegman, "Universal classes of hash functions," $J$. Comput. Syst. Sci., 1979.

[31] F. Baldimtsi, J. Camenisch, M. Dubovitskaya, A. Lysyanskaya, L. Reyzin, K. Samelin, and S. Yakoubov, "Accumulators with applications to anonymity-preserving revocation," in IEEE European Symposium on Security and Privacy (EuroS\&P), 2017.

[32] "Vitalik buterin: Ethereum will eventually achieve 1 million transactions per second." https://tinyurl.com/y8bgqsq8

[33] "Go ethereum," https://tinyurl.com/jkw5ow9

[34] "Truffle suite," https://truffleframework.com/

[35] androlo, "Solidity contracts," https://tinyurl.com/ya9s68dh

[36] "Ethereum average gas limit chart," https://tinyurl.com/yaokfvl2

[37] "Zerocoin: Solidity big number library," https://tinyurl.com/yaa34saq

[38] "Nist: Digital signature standard (dss)," https://tinyurl.com/ybjakloz

[39] "Nist: Recommendation for key management," https://tinyurl.com/ybcmxqlv

[40] R. Gennaro, S. Halevi, and T. Rabin, "Secure hash-and-sign signatures without the random oracle," in International Conference on the Theory and Application of Cryptographic Techniques (EUROCRYPT), 1999.

[41] V. Buterin, "Eip 198," https://tinyurl.com/y9mhw6jz

[42] P. B. Alex Beregszaszi, "Eip 145," https://tinyurl.com/yc4khbj8

[43] R. Gennaro, C. Gentry, and B. Parno, "Non-interactive verifiable computing: Outsourcing computation to untrusted workers," in Advances in Cryptology (CRYPTO), 2010.

[44] GnuPG, "Libgcrypt," https://tinyurl.com/yaa8m7ao 
[45] J. Baylina, "Eip 1109," https://tinyurl.com/yckxjogx

[46] "Evm opcodes and instruction reference," https://tinyurl.com/ya7o3t6c

[47] V. Buterin, "Transaction fee economics," https://tinyurl.com/y8ckvboh

[48] V. Buterin, "A simple and principled way to compute rent fees," https://tinyurl.com/y9vv6w59

[49] "Ethereum javascript api," https://tinyurl.com/y7c83nse

\section{APPENDIX}

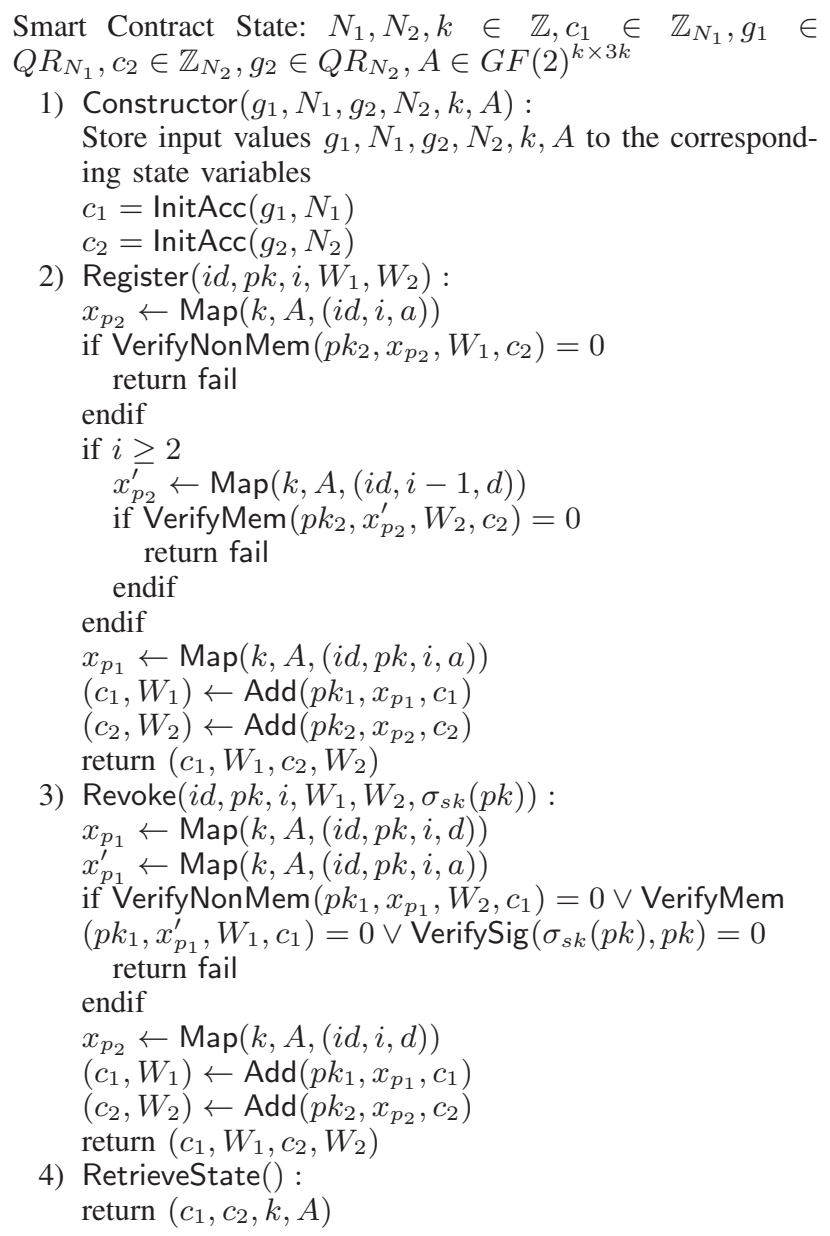

1) Constructor $\left(g_{1}, N_{1}, g_{2}, N_{2}, k, A\right)$ :

Store input values $g_{1}, N_{1}, g_{2}, N_{2}, k, A$ to the corresponding state variables

$c_{1}=\operatorname{InitAcc}\left(g_{1}, N_{1}\right)$

$c_{2}=\operatorname{Init} \operatorname{Acc}\left(g_{2}, N_{2}\right)$

2) Register $\left(i d, p k, i, W_{1}, W_{2}\right)$ :

$x_{p_{2}} \leftarrow \operatorname{Map}(k, A,(i d, i, a))$

if VerifyNonMem $\left(p k_{2}, x_{p_{2}}, W_{1}, c_{2}\right)=0$ return fail

endif

if $i \geq 2$

$x_{p_{2}}^{\prime} \leftarrow \operatorname{Map}(k, A,(i d, i-1, d))$

if $\operatorname{VerifyMem}\left(p k_{2}, x_{p_{2}}^{\prime}, W_{2}, c_{2}\right)=0$ return fail

endif

endif

$x_{p_{1}} \leftarrow \operatorname{Map}(k, A,(i d, p k, i, a))$

$\left(c_{1}, W_{1}\right) \leftarrow \operatorname{Add}\left(p k_{1}, x_{p_{1}}, c_{1}\right)$

$\left(c_{2}, W_{2}\right) \leftarrow \operatorname{Add}\left(p k_{2}, x_{p_{2}}, c_{2}\right)$

return $\left(c_{1}, W_{1}, c_{2}, W_{2}\right)$

3) $\operatorname{Revoke}\left(i d, p k, i, W_{1}, W_{2}, \sigma_{s k}(p k)\right)$ :

$x_{p_{1}} \leftarrow \operatorname{Map}(k, A,(i d, p k, i, d))$

$x_{p_{1}}^{\prime} \leftarrow \operatorname{Map}(k, A,(i d, p k, i, a))$

if VerifyNonMem $\left(p k_{1}, x_{p_{1}}, W_{2}, c_{1}\right)=0 \vee$ VerifyMem

$\left(p k_{1}, x_{p_{1}}^{\prime}, W_{1}, c_{1}\right)=0 \vee \operatorname{VerifySig}\left(\sigma_{s k}(p k), p k\right)=0$

return fail

endif

$x_{p_{2}} \leftarrow \operatorname{Map}(k, A,(i d, i, d))$

$\left(c_{1}, W_{1}\right) \leftarrow \operatorname{Add}\left(p k_{1}, x_{p_{1}}, c_{1}\right)$

$\left(c_{2}, W_{2}\right) \leftarrow \operatorname{Add}\left(p k_{2}, x_{p_{2}}, c_{2}\right)$

return $\left(c_{1}, W_{1}, c_{2}, W_{2}\right)$

4) RetrieveState () :

return $\left(c_{1}, c_{2}, k, A\right)$

Fig. 3: Pseudocode of the smart contract of the RSA-based construction. The Constructor function is executed once, during contract deployment and initializes the contract's state. The remaining functions constitute the main interface of the smart contract, i.e., registering (Register) and revoking (Revoke) an $(i d, p k)$ pair and retrieving its current state (RetrieveState).

In this section, we present figures that illustrate the pseudocode of the smart contract pertaining to each construction of Section $\mathrm{V}$. In both cases, the interface of the smart contract is comprised of four functions. The Constructor is invoked only when the contract is deployed and serves as a means to initialize its state. The Register and Revoke functions constitute the main operations of the smart contract for registering and revoking, respectively, (identity,public-key) pairs. Lastly, RetrieveState is a helper function that clients invoke to obtain
Smart Contract State: $c_{1}, c_{2}, \lambda_{1}, \lambda_{2} \in \mathbb{Z}$

1) Constructor $\left(\lambda_{1}, \lambda_{2}\right)$ :

Store input values $\lambda_{1}, \lambda_{2}$ to the corresponding state variables

$c_{1} \leftarrow \operatorname{InitAcc}\left(\lambda_{1}\right)$

$c_{2} \leftarrow \operatorname{Init} \operatorname{Acc}\left(\lambda_{2}\right)$

2) Register $\left(i d, p k, W_{2}, c_{a d d_{1}}, W_{a d d_{1}}, c_{a d d_{2}}, W_{a d d_{2}}\right)$ :

if $\operatorname{sizeof}(i d) \neq \lambda_{2} \vee$ CheckUpdate $\left(c_{2}, c_{a d d_{2}}, W_{a d d_{2}}, i d\right)$

$=0 \vee \operatorname{sizeof}(i d, p k) \neq \lambda_{1} \vee$ CheckUpdate $\left(c_{1}, c_{a d d_{1}}\right.$,

$\left.W_{a d d_{1}},(i d, p k)\right)=0 \vee \operatorname{Verify} \operatorname{NonMem}\left(c_{2}, W_{2}, i d\right)=0$ return fail

endif

$c_{1} \leftarrow c_{a d d_{1}}$

$c_{2} \leftarrow c_{a d d_{2}}$

3) Revoke $\left(i d, p k, W_{1}, \sigma_{s k}(p k), c_{d e l_{1}}, W_{\text {del }_{1}}, c_{\text {del }_{2}}, W_{\text {del }_{2}}\right)$ : if $\operatorname{sizeof}(i d) \neq \lambda_{2} \vee \operatorname{sizeof}(i d, p k) \neq \lambda_{1} \vee \operatorname{VerifyMem}\left(c_{1}\right.$, $\left.W_{1},(i d, p k)\right)=0 \vee \operatorname{VerifySig}\left(\sigma_{s k}(p k), p k\right)=$ $0 \vee$ CheckUpdate $\left(c_{1}, c_{d e l_{1}}, W_{d e l_{1}},(i d, p k)\right)=0 \vee$ CheckUpdate $\left(c_{2}, c_{d e l_{2}}, W_{d e l_{2}}, i d\right)=0$ return fail

endif

$c_{1} \leftarrow c_{d e l_{1}}$ $c_{2} \leftarrow c_{d e l_{2}}$

4) RetrieveState () :

return $\left(c_{1}, c_{2}, \lambda_{1}, \lambda_{2}\right)$

Fig. 4: Pseudocode of the smart contract in the Hash-based construction. The Constructor function is executed once, during contract deployment and initializes the contract's state. The remaining functions constitute the main interface of the smart contract, i.e., registering (Register) and revoking (Revoke) an $(i d, p k)$ pair and retrieving its current state (RetrieveState). The sizeof operator outputs the number of bits of its input.

the current state of the smart contract, e.g., via Ethereum's web3.js API ([49]). The Solidity code of the smart contracts, as well as, that of all the building blocks that we employ in our implementations, can be made available upon request.

Figure 3 illustrates the pseudocode of the RSA-based PKI of Section V-A Its state is comprised of the following: 1) two RSA accumulator values, $c_{1}$ and $\left.c_{2}, 2\right)$ the accumulators' respective RSA moduli, $N_{1}$ and $N_{2}, 3$ ) the accumulators' exponentiation bases, $g_{1}$ and $g_{2}$, which are quadratic residues modulo $N_{1}$ and $N_{2}$, respectively and, 4) the parameters of the Map procedure where, $k$, is its security parameter that, among others, determines the bit length of the primes that Map outputs ( $3 k$ bits long) and, $A$, is a randomly generated $k \times 3 k$ bit matrix.

Figure 4 illustrates the pseudocode of the Hash-based PKI of Section $\mathrm{V}$-B. Its state is comprised of the following: 1) two Hash accumulator values, $c_{1}$ and $c_{2}$ and, 2) the accumulators' respective security parameters, $\lambda_{1}$ and $\lambda_{2}$, which, essentially, determine the number of bits of the accumulators' inputs. To this end, and as illustrated in the smart contract's pseudocode, we employ a sizeof operator which, on input an arbitrary string $s$, outputs its bit length. 